\title{
THE ECMI IN TURBULENT RECONNECTING CURRENT LAYERS IN STRONG GUIDE FIELDS
}

\author{
R. A. Treumann*, and W. Baumjohann ${ }^{\dagger}$
}

\begin{abstract}
The ECMI is excited when an at least weakly relativistic electron distribution is anisotropic exhibiting higher occupations at perpendicular than parallel velocities. It requires strong magnetization and plasma dilution $\beta<1$, satisfied whenever electric fields expel electrons from some region. This is the case in planetary auroral regions, and coronae of magnetized stars like the sun. Loss-cone distributions on the global scale are one candidate for the ECMI. On the small scale, localized electric fields like in electron holes can provide the required dilution and anisotropy. They are excited by strong field-aligned currents preferentially in high density regions thus violating the dilution condition. The ECMI causes radiation trapped in the holes if the holes are sufficiently extended. Escape of radiation takes place after the hole propagates along the field into a region where the frequency exceeds the lower Xmode cut-off. Holes are, however, small-scale entities. A more promising candidate for the EMCI is spontaneous reconnection in strong guide fields along current flow. Reconnection generates ion-scale and thus meso-scale electron exhausts. In guide fields they satisfy the $\beta$-condition and produce perpendicular anisotropies (mainly electron beams) on the distribution function. Such conditions are in favor of ECMI. Since reconnection takes place in many small-scale electron filaments, large numbers of such reconnection exhausts in plasma, like in plasma undergoing turbulence, may cause high emissivities of radio waves. In sufficiently strong guide fields the escape condition is not problematic. Moreover, O-modes and higher X-harmonics can also be excited in this way. This may cause relatively weak polarizations. Spontaneous reconnection in regions of magnetically turbulent plasmas in strong magnetic fields, present for instance in extended field aligned current sheets, but also in large volumes containing magnetic turbulence that are penetrated by strong guide fields, might in this way become source regions of the ECMI and radiate in moderately strong radio waves. In particular large-scale plasmas embedded in relatively strong magnetic fields and exhibiting turbulence would in this case shine in nearly stationary radio waves of such non-thermal and non-synchrotron origin caused by the ECMI in many distributed reconnection exhausts if only a sufficiently strong large-scale magnetic guide field penetrates the plasma. Such a model will be investigated semi-quantitatively.
\end{abstract}

\footnotetext{
* Department of Geophysics, Munich University, Munich, Germany

$\dagger$ Space Research Institute, Austrian Academy of Sciences, Graz, Austria
} 
